\title{
Foot length, an accurate predictor of nasotracheal tube length in neonates
}

\author{
N D Embleton, S A Deshpande, D Scott, C Wright, D W A Milligan
}

\begin{abstract}
Background-Existing guidelines for optimal positioning of endotracheal tubes in neonates are based on scanty data and relate to measurements that are either non-linear or poorly reproducible in sick infants. Foot length can be measured simply and rapidly and is related to a number of external body measurements.

Objectives-To evaluate the relation of foot length to nasotracheal length in direct measurements at post mortem examinations, and then compare its clinical relevance with traditional weight based estimates in a randomised controlled trial.
\end{abstract}

Methods-The dimensions of the upper airway were measured at autopsy in 39 infants with median (range) postmenstrual age and birth weight of 32 (24-43) weeks and $1630(640-3530)$ g. The regression equations with $95 \%$ prediction intervals were calculated to estimate the optimal nasotracheal length from foot length. In a randomised trial, 59 neonates were nasally intubated according to foot length and body weight based estimates to assess the achievement of "optimal" and "satisfactory" tube placements.

Results-In the direct measurements of the airway at autopsy, foot length was a better predictor of nasotracheal distances $\left(r^{2}=0.79\right)$ than body weight, gestational age, and head circumference $\left(r^{2}=0.67\right.$, 0.58 , and 0.60 respectively). Measurement of foot length was easy and highly reproducible. In the randomised controlled trial, there were no significant differences between the foot length and body weight based estimates in the rates of optimal $(44 \% v 56 \%)$ and satisfactory $(83 \% v 72 \%)$ endotracheal tube placements.

Newcastle Neonatal Service, Royal Victoria Infirmary, Queen

Victoria Road,

Newcastle upon Tyne

NE1 4LP, UK

N D Embleton

D W A Milligan

Department of

Pathology

D Scott

C Wright

Royal Shrewsbury Hospital, Mytton Oak Road, Shrewsbury

SY3 8XQ, UK

S A Deshpande

Correspondence to: Dr Deshpande

deshpande@which.net

Accepted 26 January 2001

Accurate positioning of the endotracheal tube (ETT) in mechanically ventilated neonates is essential for optimal ventilation, yet as many as half of intubated babies may have atelectasis secondary to endobronchial placement of tubes. ${ }^{1}$ Indeed, malposition of the ETT is the most common unpredicted radiographic finding necessitating intervention. ${ }^{2}$ The accepted optimum position for the tip of the ETT is in the midtrachea, and a number of nomograms are available to predict this depth from external body measurements such as weight, head circumference, crown-heel or crown-rump length. ${ }^{13-5}$ However, many of these studies have their limitations. The original cadaveric work ${ }^{3}$ included only 15 infants, only one of these being less than $1000 \mathrm{~g}$ in weight. Today, a large number of infants admitted to the neonatal intensive care unit weigh less than $1000 \mathrm{~g}$. Although studies show crown-rump ${ }^{5}$ or crownheel lengths ${ }^{6}$ to be the best predictors of midtracheal distance, they are difficult to measure accurately in sick babies and lack reproducibility. Weight is easy to obtain accurately but it is a non-linear measurement, and predictions based on it may be misleading in oedematous or growth retarded infants. Head circumference is related more to brain mass than body length and may be misleading in infants with cephalohaematoma, caput succedaneum, and microcephaly or macrocephaly.

It has been shown that the birth weights and crown-rump and crown-heel lengths of neonates, particularly premature babies, can be accurately estimated from measurement of their foot lengths, and that the latter measurement can be made simply, rapidly, and safely even in critically ill neonates. ${ }^{7}$ We hypothesised that foot length may provide a simple and accurate prediction of optimal ETT length. We evaluated the usefulness of this measurement in estimating the naris to midtracheal length in neonates during direct measurements of the upper airway at autopsy, and have tested its clinical relevance against the traditional weight derived estimates in a randomised clinical trial.

\section{Patients and methods}

OVERVIEW

We measured the naris to midtracheal length at autopsy in 39 neonatal deaths and nonmacerated still births, and investigated its relation to external body measurements (post mortem study). Foot length was the best variable for prediction of naris to midtracheal length. We then compared the accuracy and reliability of the estimation of ETT length from foot length against the body weight in a prospective randomised controlled trial.
POST MORTEM STUDY

Data were collected on the dimensions of the upper airway and external body measurements at autopsy in 39 neonatal deaths and nongiven consent for post mortem examination. macerated fresh stillbirths; the parents had 
We excluded babies with fetal hydrops, congenital malformations, or chromosomal anomalies. Post mortem body weight, occipitofrontal head circumference (OFC), crownrump length (CRL), shoulder-umbilicus length (SUL), sternal notch-umbilicus length (STUL), and foot length were measured before autopsy dissection.

The upper airway dimensions were measured with the trachea in situ. After removal of the sternum, the left lung and heart were displaced to the right. The carina was identified and incised. A firm graduated $3 \mathrm{~mm}$ catheter was then inserted and passed upwards through the trachea, larynx, pharynx, and nasal cavity until the tip of the tube appeared at the nostril. The nose-carina (NC) length was then measured using the gradations on the tube. The vocal cord-carina (VC) distance was measured directly after removal of the thoracic organs and opening of the posterior wall of the larynx and trachea. The naris-midtracheal length (NMTL) was calculated as $\mathrm{NC}-(0.5 \times$ VC). All these lengths were measured in $\mathrm{mm}$.

\section{Statistical analysis}

Linear regression analysis was used to investigate the relation of NMTL to potential predictors, including corrected gestational age, weight at autopsy, OFC, CRL, SUL, STUL, and foot length.

CLINICAL STUDY

Variability in foot length measurement

Interobserver variability in foot length measurement was assessed by having the same foot measured by 10 different nurses within six hours in seven infants. Intraobserver variability was evaluated by measurement of the same foot by the same observer on 10 occasions in four babies. The within subject standard deviations $\left(\zeta_{\omega}\right)$ were estimated by one way analysis of variance, and the repeatability (the difference between two measurements for the same subject for $95 \%$ pairs of observations) was calculated as $2.77 \zeta_{\omega}$.

We designed a prospective randomised clinical study to assess the accuracy of the new foot length based prediction against the traditional weight based estimates. In view of the nature of the study, local research ethics committees did not stipulate informed parental consent while granting approval for the study.

\section{Study subjects}

The study subjects were all neonates needing nasotracheal intubation by the neonatal unit staff, including those intubated by the neonatal interhospital transport team. We excluded babies who were hydropic, dysmorphic, or intubated solely by the referring paediatricians.

\section{Study design}

Randomisation was performed in advance with computer generated codes, and assignments were inserted in sealed consecutively numbered opaque envelopes. The randomisation code was revealed when an eligible infant was considered for nasotracheal intubation. If nasotracheal intubation was unsuccessful, then the envelope was discarded.
In infants assigned to "weight", the birth weight or the most recent weight (if greater than the birth weight) were used to determine the predicted nasotracheal tube length. For infants drawing "foot length", this was defined as the distance from the tip of the heel to the tip of the great toe, and this length was measured using a plastic ruler. The foot length was rounded to the nearest $0.5 \mathrm{~cm}$ mark. The estimated ETT length was then read off the appropriate charts. For both groups, up to 0.5 $\mathrm{cm}$ was added to the predicted ETT length to allow for the fixation of the tube. The infant was then nasally intubated using the ETT appropriate to the infant's weight and naris size.

The nasotracheal tubes were fixed using the method described by Gregory. ${ }^{8}$ The equality of air entry was checked by auscultation, and radiological confirmation of the tip of the ETT was obtained as soon as possible. The chest radiograph after intubation was obtained with the infant lying directly on the roentgenogram plate with the head in the midline and the neck in the neutral position. The beam was centred on the sternal angle.

\section{Outcome measures}

The primary outcome measure was the achievement of "optimal midtracheal" ETT position defined as the tip of the tube at the body of the first thoracic vertebra. ${ }^{9}$ Secondary outcome measures were (a) achievement of "satisfactory" ETT position defined as the tip of the tube located within the trachea from between the medial ends of the clavicle to at least $1.5 \mathrm{~cm}$ above the carina, and $(b)$ the need for reintubation. If the ETT position was considered unsatisfactory, needing adjustment or reintubation, this was considered a failure.

\section{Statistical analysis}

Based on previous data, we assumed that body weight based estimates will achieve optimal ETT placement in $60 \%$. To enable a $30 \%$ difference in the achievement of optimal ETT placement with $80 \%$ power at the $5 \%$ significance level, we needed 32 infants in each group. Statistical analysis was performed with STATA 5.0 for Macintosh (Stata Corporation, College Station, Texas, USA). The basic characteristics of the two groups were compared by Mann-Whitney $U$ test for continuous and $\chi^{2}$ test for the categorical variables. The outcome data were compared by the two tailed $\chi^{2}$ test with Fisher's exact modification where necessary.

\section{Results}

POST MORTEM STUDY

The median (range) postmenstrual age and birth weight of the infants were 32 (24-43) weeks and 1630 (640-3530) g respectively. Ten infants were less than 30 weeks gestation, and seven weighed less than $1000 \mathrm{~g}$. Table 1 shows the regression equations and residual SDs for each of the anthropometric measurements (weight, OFC, CRL, SUL, and foot length) and gestational age with NMTL. Foot length was the best single anthropometric measure for 
Table 1 Regression equations for naris to midtracheal distance in $\mathrm{mm}$ derived from gestational age (Gest), body weight (Wt), Occipitofrontal head circumference (OFC), crown-rump length (CRL), shoulder to umbilicus length (SUL), and foot length (FL)

\begin{tabular}{lll}
\hline Measurement & Regression equation & Residual SD \\
\hline Gest (weeks) & $25.7+1.97 \mathrm{Gest}$ & 8.89 \\
Wt $(\mathrm{g})$ & $64.3+0.01 \mathrm{Wt}$ & 7.80 \\
OFC (mm) & $16.7+0.26 \mathrm{OFC}$ & 8.67 \\
CRL $(\mathrm{mm})$ & $2.20+0.30 \mathrm{CRL}$ & 6.56 \\
SUL $(\mathrm{mm})$ & $23.4+0.49 \mathrm{SUL}$ & 8.39 \\
FL $(\mathrm{mm})$ & $24.4+1.06 \mathrm{FL}$ & 6.42 \\
\hline
\end{tabular}

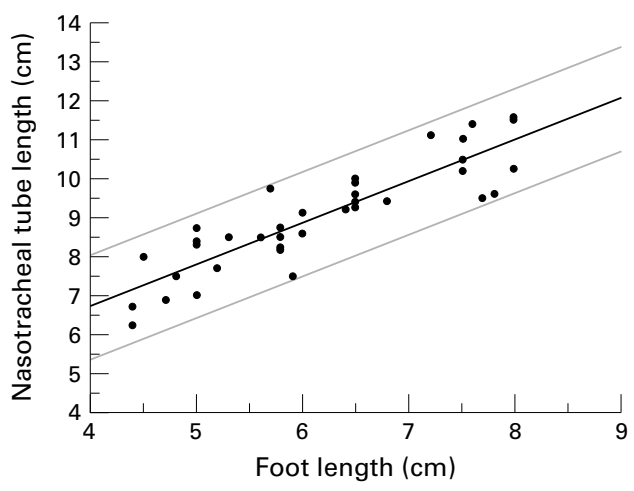

Figure 1 Graph for prediction of optimal endotracheal tube (ETT) length (with 95\% prediction intervals) for nasotracheal intubation based on measurement of foot length. (Add $0.5 \mathrm{~cm}$ to the measured ETT length if the tube is sutured to a tape.)

predicting the NMTL as well as other upper airway dimensions such as NC and VC (the regression data for the latter not shown) followed closely by the CRL. Figure 1 shows the graph for estimating the optimal ETT length from foot length with $95 \%$ prediction intervals. We then used this graph to estimate nasotracheal tube length for intubation in the randomised trial.

\section{CLINICAL STUDY}

Measurement error

The within subject standard deviation in the interobserver study was $1.47 \mathrm{~mm}$, giving a repeatability of $4 \mathrm{~mm}$. For the intraobserver variation, the within subject standard deviation was $0.9 \mathrm{~mm}$ with a repeatability of $2.5 \mathrm{~mm}$.

Randomised controlled trial of nasotracheal intubation in neonates

Of the 72 infants randomised in the trial, 13 could not be successfully nasally intubated. Confirmatory chest radiographs after intubation were unavailable in a further four (two in

Table 2 Baseline characteristics of study infants

\begin{tabular}{|c|c|c|}
\hline & \multicolumn{2}{|c|}{ Method of estimating nasotracheal tube length } \\
\hline & Body weight $(n=36)$ & Foot length $(n=36)$ \\
\hline $\begin{array}{l}\text { Unsuccessful nasal intubation or confirmatory } \\
\text { radiograph unavailable }\end{array}$ & 11 & 6 \\
\hline $\begin{array}{l}\text { Infants successfully randomised with } x \text { ray } \\
\text { confirmation of ETT position }\end{array}$ & 25 & 30 \\
\hline Boys:girls & $14: 11$ & $11: 19$ \\
\hline Birth weight $(\mathrm{g})^{\star}$ & $1210(990-1770)$ & $1467(915-1790)$ \\
\hline Gestational age (weeks) ${ }^{\star}$ & $30(27-36)$ & $30.5(27-32)$ \\
\hline Age at intubation (days) & $1(0-1)$ & $0(0-3)$ \\
\hline Weight at intubation $(\mathrm{g})^{\star}$ & $1210(990-1770)$ & $1467(915-1790)$ \\
\hline Number of infants $<1000 \mathrm{~g}$ & 9 & 8 \\
\hline
\end{tabular}

^Median (interquartile range).

ETT, Endotracheal tube.
Table 3 Endotracheal tube (ETT) placements

\begin{tabular}{llll}
\hline & Body weight & Foot length & p Value \\
\hline Number of infants & 25 & 30 & \\
Satisfactory ETT & $18(72)$ & $25(83)$ & $0.49^{\star}$ \\
Optimal ETT & $14(56)$ & $13(44)$ & $0.50^{\star}$ \\
Unsatisfactory ETT & & & \\
$\quad$ High & 5 & 4 & $0.76 \dagger$ \\
Low & 2 & 1 & $0.86 \dagger$
\end{tabular}

Values in parentheses are percentages of successful intubations with $x$ ray confirmation of ETT position.

${ }^{\star} \chi^{2}$ test with Yates' continuity correction.

†Fisher's exact test.

each group) infants, although all these infants were successfully ventilated and none required reintubation. The baseline characteristics were similar for the two groups of randomised infants (table 2). Seventeen infants weighed less than $1000 \mathrm{~g}$ at intubation.

There were no statistically significant differences in the rates of achievement of optimal and satisfactory placements of the ETTs between the two groups (table 3). In three infants from the body weight group and two from the foot length group, the ETTs needed repositioning. Of 10 infants in whom the ETT tip was located at vertebral bodies C6-8, one was between, and nine were above, the medial ends of the clavicles. Of 32 infants in whom the ETT tip was between the first and second thoracic vertebrae, 19 were between the medial ends of the clavicles, with the remainder just below it. Of the five ETT tips at T4-5, two were in the low trachea (but safe) and three were at or below the carina.

\section{Discussion}

Our direct measurements of the airway dimensions in post mortem examinations showed that foot length was the best predictor of narismidtracheal length. In a subsequent randomised clinical trial against traditional body weight based estimates, foot length proved an accurate, reproducible, and easy to measure alternative predictor for estimating optimal nasotracheal tube length for neonatal intubation.

\section{STRENGTHS}

Our hypothesis that foot length provides an accurate prediction of naris-midtracheal length in babies was confirmed by direct measurements of the airway at autopsy. We excluded dysmorphic infants from both studies as the tracheal length in such infants may be shorter than normal. ${ }^{10}$ We also precisely defined the optimum position for the tip of the ETT in the midtrachea as corresponding radiologically to the first thoracic vertebra. ${ }^{9}$ We included a pragmatic measure of satisfactory ETT length because babies who may not have an ideally placed ETT can still be successfully ventilated. Flexion of the head decreases the naris to carina distance (a risk for bronchial intubation) by up to $1 \mathrm{~cm}$ in infants under $1000 \mathrm{~g}$ and up to $1.6 \mathrm{~cm}$ in bigger infants. ${ }^{5}$ We therefore chose the distance of $1.5 \mathrm{~cm}$ above the carina as a safe position. Although a number of measurements other than body weight have been suggested for estimation of optimal depth of ETT placement 
in babies, ${ }^{611-15}$ they have not been evaluated in randomised controlled trials.

\section{LIMITATIONS}

We based the predictive equations on the data collected at autopsy. In vivo traction from the diaphragm increases the length of the trachea during inspiration. Loss of this traction effect after death results in a shorter trachea. We attempted to limit this effect by measuring tracheal lengths in situ before its attachments were severed. Observations at bronchoscopy in live infants, ${ }^{16}$ and radiological evaluations ${ }^{6}{ }^{12}$ have been used to provide in vivo airway dimensions, but these studies have not included small premature infants limiting their application to current neonatal practice.

We did not use a specially designed foot gauge as used by James and others, ${ }^{7}$ but found very little intraobserver and interobserver variability in the measurement of foot length using simple plastic rulers. We rounded off the foot length measurement to the nearest $0.5 \mathrm{~cm}$ mark, as the repeatability of this measurement in clinical practice was $4 \mathrm{~mm}$. As our units use the nasotracheal route for babies requiring medium to long term ventilation, we calculated the nasotracheal tube lengths. Our results are not applicable to the oral route.

\section{STRENGTHS AND WEAKNESSES IN RELATION TO} OTHER STUDIES

Although attempts have been made in recent years to locate the ETT tip sonographically at the time of intubation ${ }^{17}$ or by using a fibre optic light ${ }^{18}$ or magnetic ring embedded in the ETT tube (TRACH MATE) ${ }^{19}$ such equipment is either not widely available or adds complexity. Optimal ETT length is therefore commonly estimated from nomograms based on some external body measurement. The traditionally used weight or head circumference based graphs are derived from anatomic measurements of excised tissues at autopsies of mainly term infants, ${ }^{34}$ limiting their relevance to small preterm babies. Although Rotschild and colleagues ${ }^{5}$ provided regression equations for optimal ETT lengths, these require measurements of crown-heel and crown-rump lengths, which are difficult to obtain accurately and without disturbing a critically ill ventilated baby. Shukla and colleagues ${ }^{13}$ suggested a rapid method for estimating orotracheal and nasotracheal tube lengths from nasal-tragus and sternal lengths. In a prospective study, they reported achievement of a perfect ETT tube position in 48 of 50 infants using their predictors. However, this was a non-randomised study and no definition of perfect ETT position was provided. Our study shows that, despite their excellent performance in post mortem studies, predictors of optimal ETT length achieve such positions in only half of the clinical intubations, with a fifth having unsatisfactory tube placements. Hence, a rigorous controlled clinical evaluation of newer methods of estimating optimal ETT placement is necessary.
EVALUATION OF THE STUDY: POSSIBLE

MECHANISMS AND IMPLICATIONS FOR CLINICIANS Foot length has been shown to be related positively to gestational age a $^{21}$ and length in fetuses, ${ }^{22}$ and various indices of body size in both large for dates and appropriately grown babies of all gestational ages between 26 and 42 weeks. ${ }^{7}$ In the latter study ${ }^{7}$ there were particularly pronounced correlations of foot length with body weight $(r=0.95)$ and crown-heel length $(r=0.96)$ in premature babies. As tracheal length is a linear body measurement, it is not surprising that it correlates more closely with linear measures such as foot length or crown-rump length than body weight or head circumference.

Unlike the illuminated or magnetic ring embedded ETTs, use of foot length for prediction of ETT length does require radiographic confirmation of the tube position, necessitating exposure to ionising radiation every time the location of the tube is called into question. Measurement of foot length, however, does not require any additional equipment apart from a widely available transparent plastic ruler, and can be performed easily and reproducibly even in very unstable babies. Our clinical trial shows that foot length provides an alternative, reliable, and accurate method for determining the optimal ETT length, particularly in sick unstable infants.

We wish to thank our medical and nursing colleagues and also Drs R Thiagarajan and R Wariyar for their assistance with the Drs R
study.

1 Kuhns L, Poznanski A. Endotracheal tube position in neonates. F Pediatr 1971;78:991-6.

2 Hauser GJ, Pollock MM, Sivit CJ. Routine chest radiographs in paediatric intensive care: a prospective study. Pediatrics 1989;83:465-70.

3 Coldiron J. Estimation of nasotracheal tube length in neonates. Pediatrics 1968;41:823-8.

4 Butz RO. Length and cross-section growth patterns in the human trachea. Pediatrics 1968;42:336-41.

5 Rotschild A, Chitayat D, Puterman M, et al. Optimal positioning of endotracheal tubes for ventilation of preterm infants. American fournal of Diseases in Children 1991;145:1007-12

6 Loew A, Thibeault DW. A new and safe method to control the depth of endotracheal intubation in neonates. F Pediatr 1974;54:506-8.

7 James D, Dryburgh E, Chiswick M. Foot length: a new and potentially useful measurement in the neonate. Arch Dis Child 1979;54:226-30.

8 Gregory GA. Respiratory care of newborn infants. Pediatr Clin North Am 1972;19:311-24.

9 Blayney MP, Logan DR. First thoracic vertebral body as reference for endotracheal tube placement. Arch Dis Child Featal Neonatal Ed 1994;71:F32-5.

10 Wells TR, Wells AL, Galvis DA, et al. Diagnostic aspects and syndromal associations of short trachea with bronchial intubation. Am F Dis Child 1990;144:1369-71.

11 Lingle PA. Sonographic verification of endotracheal tube position in neonates: a modified technique. 7 Clin Ultrasound 1988;16:605-9.

12 Mattila MAK, Heikel PE, Suutarinen T, et al. Estimation of a suitable nasotracheal tube length for infants and children. Acta Anaesthesiol Scand 1971;15:239-46.

13 Shukla HK, Hendricks-Munoz KD, Atakent Y, et al. Rapid estimation of insertional length of endotracheal intubation in newborn infants. F Pediatr 1997;131:561-4.

14 Tochen ML. Orotracheal intubation in the newborn infant: A method for determining depth of tube insertion. I Pediatr 1979;95:1050-1.

15 Zanardo V, Ronconi M, Bertocchi G. Correct placement of endotracheal tube by single strand fiberoptic light in prematures. Initial clinical experience. Padiatrie und Padologie 1991;26:227-8.

16 Fearon B, Whalen JS. Tracheal dimensions in the living infant. Ann Otol 1967;76:964-74

17 Slovis TL, Poland RL. Endotracheal tubes in neonates: sonographic positionings. Radiology 1986;160:262-3.

18 Heller RM, Cotton RB. Early experience with illuminated endotracheal tubes in premature and term neonates. Pediatrics 1985;75:664-6. 
19 Blayney M, Costello S, Perlman M, et al. A new system for location of endotracheal tube in preterm and term neonates. Pediatrics 1991;87:44-7.

20 Markowski B, Lawler SD. Use of early fetal tissues obtained from suction termination of pregnancy. Lancet 1977;1:186-8
21 Mercer BM, Sklar S, Shariatmadar A, et al. Fetal foot length as a predictor of gestational age. Am $\mathcal{F}$ Obstet Gynecol 1987;156:350-5

22 Pospisilova-Zuzakora V. Determination of the body length of the fetus with the aid of the length of the sole of the foot. Biologia 1962;17:49-52. 